Published in final edited form as:

JAMA Intern Med. 2016 November 01; 176(11): 1687-1692. doi:10.1001/jamainternmed.2016.5381.

\title{
Update on Medical Overuse
}

\author{
Daniel J. Morgan ${ }^{1,2,3}$, Sanket S. Dhruva ${ }^{4}$, Scott M. Wright ${ }^{5}$, and Deborah Korenstein ${ }^{6}$ \\ ${ }^{1}$ Department of Epidemiology and Public Health, University of Maryland School of Medicine \\ ${ }^{2}$ Department of Hospital Epidemiology, Veterans Affairs Maryland Health Care System, Baltimore, \\ MD \\ ${ }^{3}$ Center for Disease Dynamics, Economics and Policy (CDDEP), Washington, DC \\ ${ }^{4}$ Robert Wood Johnson Foundation Clinical Scholars Program, Yale University School of \\ Medicine, New Haven, CT and Department of Veterans Affairs West Haven, CT \\ ${ }^{5}$ Department of Medicine, Johns Hopkins University School of Medicine, Baltimore, MD \\ ${ }^{6}$ Memorial-Sloan Kettering Cancer Center, New York, NY
}

\begin{abstract}
Importance-Overuse of medical care is an increasingly recognized problem in clinical medicine.
\end{abstract}

Objective-To identify and highlight original research articles published in 2015 that are most likely to reduce overuse, organized into three categories: overuse of testing, overtreatment, and services to question. These manuscripts were reviewed and interpreted for their importance to clinical medicine.

Evidence Review-A structured review of English-language articles on PubMed published in 2015 and review of tables of contents of relevant journals to identify potential articles that related to medical overuse in adults.

Findings-We reviewed 1445 articles, of which 821 addressed overuse. Of these, 112 were deemed most relevant based on originality, methodologic quality, and number of patients potentially affected. The 10 most influential articles were selected by author consensus using the same criteria. Findings included doubling of specialty referrals and advanced imaging for simple headache (from $6.7 \%$ in 2000 to $13.9 \%$ in 2010), unnecessary hospital admission for low-risk syncope often leading to adverse events, and overly frequent screening colonoscopy for $34 \%$ of patients. Overtreatment was common with one in four patients with atrial fibrillation at low risk for thromboembolism receiving anticoagulation, $94 \%$ of testosterone replacement being off guideline recommendations, $91 \%$ of patients restarting opioids after overdose, and $61 \%$ of diabetics being treated to potentially harmfully low HbA1C levels $(<7 \%)$. New findings suggested that testing for $C$. difficile with molecular assays, cyclobenzaprine and oxycodone/acetaminophen for acute low back pain, and serial follow-up of benign thyroid nodules should be questioned.

Conclusions and Relevance-The number of articles on overuse nearly doubled from 2014 to 2015. This review promotes reflection on the top 10 articles and may lead to questioning other non-evidence based practices. 


\section{Keywords}

Overuse; overdiagnosis; overtreatment; deimplementation

\section{Introduction}

Over three years of updates on the burgeoning area of medical overuse, ${ }^{1,2}$ the study of this topic has evolved with standardization of terminology and publication of research agendas related to the care of both adults and children., ${ }^{3,4}$ Overuse is defined as care in which potential harms outweigh potential benefits. ${ }^{3,5,6}$ Overuse encompasses different problems including the use of diagnostic and screening tests that are not needed or risky, use of therapeutic procedures or medications where harms outweigh benefits, and use of overly intensive or expensive healthcare settings. Overuse can be subdivided into overuse of testing, which can lead to overdiagnosis of disease that would never cause symptoms, and overtreatment, composed of the provision of therapeutics that are not indicated or otherwise inappropriate and treatment of overdiagnosed disease. ${ }^{3,7}$

The literature on overuse has focused on clarifying the benefits and harms of clinical services or approaches, the prevalence of use of services without benefit, and understanding factors that lead to overuse. All three lines of investigation are growing despite inherent challenges related to defining benefits and harms for individual patients and the complex motivations for providing or seeking care. Recent scholarship has attempted to uncover and explain some of the drivers of overuse; these include patient tendencies to overestimate benefits and underestimates harms associated with care ${ }^{8}$, and physicians desires to provide comfort and reassurance - both for patients and themselves. ${ }^{9}$

The present article examines and describes 10 of the most important studies published in 2015 that represent medical overuse related to the care of adult patients.

\section{Literature Search and Article Selection Process}

We selected articles through a structured review of studies published in 2015 in PubMed with the Medical Subject Headings term "health services misuse" or with any of the following words in the title: overuse, overtreatment, overdiagnosis, inappropriate, and unnecessary. In EMBASE, we performed a search using the same terms as in PubMed with the additional Emtree term "unnecessary procedure". We excluded articles with "overuse injury" or "overuse injuries" in the title. Searches were limited to human studies and the English language. One of 3 authors (D.J.M., S.S.D., and D.K.) reviewed all titles for relevance to medical overuse. One of the same 3 authors reviewed all 2015 titles from 10 major medical journals (see Figure) and read abstracts and full journal articles for those of potential relevance based on the Preferred Reporting Items for Systematic Reviews and Metaanalyses guidelines. ${ }^{10}$

The structured review identified 1445 articles, 821 of which addressed medical overuse. After excluding 41 pediatric articles, 168 editorials or opinion pieces, 28 letters without data, 29 case reports or review articles, we reviewed 555 articles. Of these, $112(20.1 \%)$ 
were ranked as most relevant by at least 1 of the 3 authors based on quality of methods, magnitude of clinical effect, and number of patients potentially affected (Figure). Using the same criteria, all authors rated these 112 articles, 39 of which were highest rated. The 10 most relevant studies were selected by consensus among all authors and are highlighted in this article, organized into the categories of overuse of testing, overtreatment, and services to question.

\section{Articles}

\section{Overuse of testing}

\section{Ambulatory patients with low-risk headache receive unnecessary imaging and referrals ${ }^{11}$}

Background: Headache is very prevalent, with approximately $15 \%$ of adults reporting headache within a 3-month period. ${ }^{12}$ Almost all headaches are benign. Clinical criteria are recommended for identifying the small subset of patients with acute headache in whom imaging or specialist referral is indicated ${ }^{13}$ (ref ICSI) and an American College of Radiology Choosing Wisely ${ }^{\circledR}$ item recommends against imaging in patients with uncomplicated headache. ${ }^{14}$ Overuse of these services has not been well studied.

Results: A retrospective cohort study of 9,362 patients used representative 1999-2010 US data from the National Ambulatory Medical Care Survey and the National Hospital Ambulatory Medical Care Survey to assess services provided to patients seen in the office or the emergency department with headache and without trauma or high-risk clinical features. Use of advanced imaging nearly doubled over time: $6.7 \%$ of patients received head CT or MRI in 1999-2000 compared with 13.9\% in 2009-10 ( $\mathrm{P}<0.001)$. There was a similar rise in specialty referrals $(6.9 \%$ to $13.2 \%, \mathrm{P}=0.005)$. The frequency of lifestyle modification counseling declined over time from $23.5 \%$ in $1999-2000$ to $18.5 \%$ in $2009-10(\mathrm{P}=0.041)$, while use of opioids remained constant. Adjustment for confounders did not meaningfully change these results.

Implications: Overuse of advanced imaging and referral is common in ambulatory patients with headache and increased through 2010 (although rates decreased slightly by 2013). ${ }^{15}$ Clinicians should familiarize themselves with the indications for more aggressive care of these patients to avoid overuse. Conservative management, including behavior change and watchful waiting, is thought to be the most reasonable approach.

\section{Unnecessary hospitalization is common in patients with low-risk syncope and is associated with unnecessary testing and harm ${ }^{16}$}

Background: Syncope is common; it often leads to unnecessary hospitalization and lowyield diagnostic testing. ${ }^{17}$ The scope of inappropriate syncope hospitalization and the associated harms have not been well described.

Results: A retrospective chart review evaluated testing and outcomes in 72 patients hospitalized at a single academic medical center with low-risk syncope, a condition for which guidelines do not recommend hospitalization. These admissions represented $34 \%$ of 
all syncope admissions. Low-risk syncope patients received a mean of 10.8 tests, which commonly included head CT (88\%), head MRI (19\%), echocardiography (64\%), telemetry monitoring (93\%), and multiple laboratory tests. Nine patients (13\%) had adverse events during hospitalization. Nearly one-third of patients had incidental findings, leading to performance of or recommendation for further evaluations in a majority. Few patients (7\%), had incidental findings with the potential to result in clinical benefit.

Implications: Hospitalization for low-risk syncope is common and is associated with unnecessary testing with accordant patient harm from adverse events and workup of incidental findings. These findings are consistent with a 2015 survey study of US hospitalists in which $83 \%$ recommended unnecessary tests in response to a vignette about a patient with syncope. ${ }^{9}$ Physicians should be mindful of the potential harms associated with hospitalizing and working up patients with low-risk syncope.

\section{Screening colonoscopy at shorter-than-recommended intervals is common and varies by region ${ }^{18}$}

Background: Colorectal cancer is the second leading cause of cancer death in the US. While screening has been shown to reduce mortality, overall screening rates remain low at $60-65 \%$. The timing of follow-up colonoscopy among previously screened patients has not been well studied, and could represent overuse if the screening interval were shorter than recommended.

Results: A retrospective study evaluated follow-up testing recommendations in 1455 patients with no cancer history who underwent colonoscopy at one of 25 Veterans Affairs hospitals. Compared to a multispecialty guideline, the follow-up interval was shorter than recommended in one-third (34\%) of patients and was longer than recommended in only $2 \%$. Short interval follow-up recommendation was associated with finding hyperplastic polyps on the index study (OR, 3.1 [CI, 1.7 to 5.8]), and region (Northeast region OR 5.4 [CI, 2.1 to 13.8]).

Implications: These findings are similar to those of a smaller 2015 study that found 69\% of 871 follow-up screening or surveillance colonoscopies were performed at least 1 year sooner than recommended by guidelines. That study also noted wide variation among individual clinicians within a multispecialty group practice. Given the potential harms associated with colonoscopy and the importance of improving access to screening for large numbers of unscreened patients, overuse of colonoscopy is a public health problem. Primary care clinicians and gastroenterologists need to partner to secure follow-up colonoscopies at the appropriate time but not sooner.

\section{Overtreatment}

\section{Young, healthy patients may receive anticoagulation even when they should not $^{19}$}

Background: The $\mathrm{CHADS}_{2}$ and $\mathrm{CHA}_{2} \mathrm{DS}_{2}$-VASc scores provide guidance on which patients with atrial fibrillation should receive oral anticoagulants for the prevention of 
thromboembolism. Guidelines state that it is reasonable to avoid anticoagulation in patients with non-valvular atrial fibrillation without any risk factors for stroke (i.e. a score of zero). ${ }^{20}$

Results: This study analyzed the National Cardiovascular Data Registry's Practice Innovation and Clinical Excellence Registry of over 10,000 atrial fibrillation patients aged $<60$ years old with no structural heart disease and with $\mathrm{CHADS}_{2}$ and $\mathrm{CHA}_{2} \mathrm{DS}_{2}$-scores of 0 . In the two cohorts, $2561(23.3 \%)$ and 1787 (26.6\%) patients, respectively, were prescribed an oral anticoagulant. In both cohorts, patients prescribed oral anticoagulants were older (mean age 51), had a higher body mass index, were more frequently either insured by Medicare or uninsured, and were less likely to have paroxysmal atrial fibrillation. There were also regional differences in oral anticoagulant prescriptions.

Implications: Unless patients with atrial fibrillation and a CHADS 2 or $\mathrm{CHA}_{2} \mathrm{DS}_{2}-\mathrm{VASc}$ score of 0 have other reasons for anticoagulation, these medications should be avoided. In these patients, bleeding risks outweigh potential benefits.

\section{Testosterone replacement is mostly prescribed inappropriately 21}

Background: Testosterone prescriptions have increased substantially in the past decade, with nearly $4 \%$ of men in their 60 s taking androgen replacement. ${ }^{22}$ This therapy may be associated with increased risk of cardiovascular disease. ${ }^{23}$ Male androgen deficiency is diagnosed by signs, symptoms, and two low morning testosterone levels. Guidelines recommend against androgen therapy in patients with either an elevated hematocrit or elevated PSA without further evaluation.

Results: Of 111,631 men in the Veterans Health Administration who first received testosterone between 2009 and 2012, only 5.4\% had a diagnosis of androgen deficiency established by two low morning testosterone levels; $16.5 \%$ had no testosterone levels checked. Over $15 \%$ did not have a baseline hematocrit checked and $24 \%$ of patients did not have a baseline PSA checked. Nearly $13 \%$ of patients had a relative contraindication (obstructive sleep apnea, hematocrit $>50 \%$, PSA $>4.0 \mathrm{ng} / \mathrm{dL}$ ), and $1.4 \%$ had prostate cancer which is an absolute contraindication.

Implications: Almost 95\% of testosterone replacement is prescribed in ways that are inconsistent with guideline recommendations. Clinicians should limit testosterone replacement to patients with signs and symptoms of deficiency and in whom there are no contraindications.

\section{Patients continue receiving opioids even after overdose 24}

Background: Prescription opioid use has increased substantially in recent years, with 259 million such prescriptions written in 2012 . Over 420,000 patients present annually to the emergency department because of nonmedical prescription opioid use. ${ }^{25}$

Results: A retrospective cohort study of a large US health insurer evaluated prescription patterns in patients with nonfatal opioid overdoses during long-term therapy. Within 10 months of overdose, $91 \%$ of these patients again received opioid prescriptions, $1 / 3$ received 
high-dose opioids, and 58\% received a benzodiazepine. Repeat overdose occurred in $7 \%$ of patients; opioid discontinuation was associated with a lower risk of subsequent overdose.

Implications: Prescribing guidelines state that patients with nonfatal overdoses should have opioids discontinued when possible. ${ }^{25}$ Emergency department presentation or hospitalization for nonfatal opioid overdose is an opportunity to identify and refer patients with opioid abuse for substance treatment to discontinue opioid prescriptions.

\section{Tight glycemic control is rarely de-intensified in older adults with diabetes mellitus $^{26}$}

Background: For many older adults with type 2 diabetes, attempts to achieve intensive glycemic control will lead to net harm such as hypoglycemia. ${ }^{27}$ A 2012 American Diabetes Association and American Geriatrics Society consensus statement endorses less aggressive glycemic targets in older patients with limited life expectancy, in whom tight glycemic control is unlikely to be beneficial. ${ }^{28}$

Results: In a study of 1288 adults 65 or older (mean age 73.2 years), from the National Health and Nutrition Examination Survey, with diabetes and a hemoglobin A1c (HbA1c) measurement, $61.5 \%$ had an $\mathrm{HbA1c}$ less than $7 \%$. Of these patients, $54.9 \%$ were treated with either insulin or sulfonylureas, and $4.0 \%$ received both. Among older adults with complex/intermediate health and very complex/poor health, $63.0 \%$ and $56.4 \%$, respectively, had an $\mathrm{HbA} 1 \mathrm{c}$ less than $7 \%$, and $44.9 \%$ and $37.9 \%$, respectively, had an HbA1c less than $6.5 \%$. The proportion of older adults with a HbA1c of less than 7 remained stable from 2000 to 2010 .

Implications: A large proportion of older adults with diabetes have intensive glycemic control, including many patients with poor health. This aggressive therapy likely results in harms exceeding benefits. These findings are consistent with another 2015 study suggesting overtreatment, in which de-intensification of diabetes therapy was rare in patients with low HbA1c levels. ${ }^{29}$

\section{Services to question}

\section{The harms of oxycodone/acetaminophen and cyclobenzaprine outweigh benefits in patients with acute low back pain treated with naproxen ${ }^{30}$}

Background: Back pain is among the most common complaints leading to ambulatory visits. ${ }^{31}$ It also results in over 2.5 million annual emergency department (ED) visits in the US. ${ }^{32}$ Nearly $2 / 3$ of patients seen in the ED for acute low back pain are prescribed opioids, over $40 \%$ are prescribed muscle relaxants ${ }^{32}$, and most are prescribed combinations of therapies. However, the efficacy of these therapies is not clear.

Results: A randomized, controlled trial of 323 patients presenting to the ED with acute low back pain without inciting trauma or radicular symptoms compared functional and pain outcomes at 1 week. Patients were randomized to treatment with placebo, cyclobenzaprine 5 $\mathrm{mg}$, or oxycodone/acetaminophen $325 / 5 \mathrm{mg}$, in addition to naproxen, and instructed to take 1 to 2 pills of study drug every 8 hours as needed. At one week, the groups did not differ 
with regard to functional status (the primary outcome), pain, use of health care resources, or return to work or usual activity. Adverse events, such as drowsiness, dizziness and nausea, were more common in the oxycodone/acetaminophen (number needed to harm, NNH 5) and cyclobenzaprine (NNH 8) groups compared to the placebo group.

Implications: Treating ED patients with acute musculoskeletal low back pain with either oxycodone/acetaminophen or cyclobenzaprine appears to result in harm, and is without apparent benefit beyond that which is achieved with naproxen alone. While the study did not address patients seen in primary care practices, it seems prudent to avoid these therapies in this population as well; insofar as patients presenting to the office may have less severe complaints than ED patients, they may be less likely to benefit from add-on therapies and equally likely to experience harms.

\section{Frequent overdiagnosis of Clostridium difficile infection with molecular testing 33}

Background: Clostridium difficile infection (CDI) is epidemic worldwide. CDI testing has evolved from a simple toxin test to the ultrasensitive polymerase chain reaction (PCR) testing in many laboratories. Many more patients are colonized than infected with $C$. difficile.

Results: Over a two-year cohort at one US medical center, 1416 hospitalized patients were tested for CDI with the toxin test only. Samples were also evaluated with PCR testing, without results being reported to clinicians. Two hundred ninety-three samples were positive by PCR, but only $131(44.7 \%)$ were positive by toxin test. Toxin -/PCR + patients had lower bacterial loads, less antibiotic exposure, and less inflammation than those who were Toxin + and PCR +. Very few patients who were toxin -/PCR + were treated, and their clinical outcomes were similar to those in patients who were negative by both tests (median 2 days diarrhea in both groups).

Implications: A PCR test detected more than twice as much $C$. difficile as the toxin method. Given that the outcomes in patients who were toxin -/ PCR + were comparable to those who were negative by both tests, PCR testing leads to overdiagnosis. Continuing testing primarily with the toxin assay, or perhaps using a multistep testing strategy, will help avoid overdiagnosis and overtreatment.

\section{Serial follow-up of benign thyroid nodules is likely unnecessary ${ }^{34}$}

Background: Thyroid nodules are present in as many as $2 / 3$ of the adult population. ${ }^{35}$ Despite growing awareness that thyroid cancer is overdiagnosed and overtreated and a paucity of evidence to inform practice, clinical practice guidelines recommend routine imaging to follow-up most nodules. ${ }^{36}$

Results: A prospective study evaluated 992 patients with benign thyroid nodules up to $4 \mathrm{~cm}$ in diameter. Over 5 years, most patients had no change in nodule number $(88.3 \%)$ or size (69.0\%). Seven patients (0.7\%) were diagnosed with thyroid cancer, among whom 3 had 
nodule growth, 3 had stable nodule size, and one was found incidentally during an unrelated thyroidectomy.

Implications: Benign thyroid nodules are unlikely to change during surveillance over 5 years, and the diagnosis of thyroid cancer is very rare. Routine follow-up testing rarely impacts care and should be questioned by individual clinicians and re-examined by organizations issuing guidelines.

\section{Conclusions}

In 2015, the literature on medical overuse continued its rapid expansion, with 821 articles identified compared to 440 in $2014 .^{2}$ The studies selected for highlighting in this manuscript emphasized: overuse of testing, referrals, and hospital admissions for low-risk syncope, uncomplicated headache, and colon cancer screening; overtreatment with respect to testosterone replacement, aggressive glycemic control among older diabetics, continuing opioid treatment following overdoses, anticoagulation of low risk younger adults with atrial fibrillation; and practices to be questioned including prescribing secondary medications beyond non-steroidal anti-inflammatories for uncomplicated acute low back pain, using PCR to detect the presence of $C$. difficile, and performing ultrasonography to follow small thyroid nodules. All of these studies have implications that could impact healthcare outcomes, safety, quality, and economics.

In careful reading of these 10 articles and review of many others from 2015, common themes and pearls emerge. The overuse of medications for which harms are likely to outweigh benefits is commonplace. Health care continues to be enthralled by high-tech innovation, including both therapies and tests. Once practice norms are established, clinicians are slow to de-implement services, even those that are found to be potentially dangerous. The small amount of data evaluating trends in overuse over time suggests an increase in overuse through 2010 without substantive improvement. However, heightened awareness of the problems associated with overuse, and advances in the overuse literature make us hopeful that we are primed for reducing overuse in the near future.

\section{Acknowledgments}

DJM had full access to all the data in the study and takes responsibility for the integrity of the data and the accuracy of the data analysis.

DJM has no COIs related to this work. Potential COIs including having served as a consultant for research studies for Welch Allyn, self-developed an educational lecture for $3 \mathrm{M}$ and received honoraria from Springer for Textbook and journal editing. He has been reimbursed for travel by multiple conferences. He receives research funding from the VA, CDC and AHRQ. All other authors report no COIs or potential COIs.

No funding organization had any role in this work.

\section{References}

1. Morgan DJ, Wright SM, Dhruva S. Update on medical overuse. JAMA Intern Med. 2015; 175(1): 120-124. [PubMed: 25365707]

2. Morgan DJ, Dhruva SS, Wright SM, Korenstein D. Update on medical practices that should be questioned in 2015. JAMA Intern Med. 2015; 175(12):1960-1964. [PubMed: 26551354] 
3. Morgan DJ, Brownlee S, Leppin AL, et al. Setting a research agenda for medical overuse. BMJ. 2015; 351:h4534. [PubMed: 26306661]

4. Coon ER, Quinonez RA, Moyer VA, Schroeder AR. Overdiagnosis: How our compulsion for diagnosis may be harming children. Pediatrics. 2014; 134(5):1013-1023. [PubMed: 25287462]

5. Welch HG, Schwartz L, Woloshin S. Overdiagnosed: Making people sick in the pursuit of health. Boston, MA: Beacon Press; 2012.

6. Chassin MR, Galvin RW. The urgent need to improve health care quality. institute of medicine national roundtable on health care quality. JAMA. 1998; 280(11):1000-1005. [PubMed: 9749483]

7. Welch HG, Passow HJ. Quantifying the benefits and harms of screening mammography. JAMA Intern Med. 2014; 174(3):448-454. [PubMed: 24380095]

8. Hoffmann TC, Del Mar C. Patients' expectations of the benefits and harms of treatments, screening, and tests: A systematic review. JAMA Intern Med. 2015; 175(2):274-286. [PubMed: 25531451]

9. Kachalia A, Berg A, Fagerlin A, et al. Overuse of testing in preoperative evaluation and syncope: A survey of hospitalists. Ann Intern Med. 2015; 162(2):100-108. [PubMed: 25599349]

10. Moher D, Liberati A, Tetzlaff J, Altman DG. PRISMA Group. Preferred reporting items for systematic reviews and meta-analyses: The PRISMA statement. Int J Surg. 2010; 8(5):336-341. [PubMed: 20171303]

11. Mafi JN, Edwards ST, Pedersen NP, Davis RB, McCarthy EP, Landon BE. Trends in the ambulatory management of headache: Analysis of NAMCS and NHAMCS data 1999-2010. J Gen Intern Med. 2015; 30(5):548-555. [PubMed: 25567755]

12. Centers for Disease Control and Prevention (CDC). [Accessed 06/02/2016/] UPDATED: Table 47. severe headache or migraine, low back pain, and neck pain among adults aged 18 and over, by selected characteristics: United states, selected years 1997-2012. http://www.cdc.gov/nchs/ data/hus/2012/047.pdf

13. Beithon J, Gallenberg M, Johnson K. , et al. Diagnosis and treatment of headache. 2013.

14. American College of Radiology. [Accessed 06/02/2016/] Don't do imaging for uncomplicated headache. http://www.choosingwisely.org/clinician-lists/american-college-radiology-imaging-foruncomplicated-headache/

15. Rosenberg A, Agiro A, Gottlieb M, et al. Early trends among seven recommendations from the choosing wisely campaign. JAMA Intern Med. 2015; 175(12):1913-1920. [PubMed: 26457643]

16. Canzoniero JV, Afshar E, Hedian H, Koch C, Morgan DJ. Unnecessary hospitalization and related harm for patients with low-risk syncope. JAMA Intern Med. 2015; 175(6):1065-1067. [PubMed: 25894921]

17. Mendu ML, McAvay G, Lampert R, Stoehr J, Tinetti ME. Yield of diagnostic tests in evaluating syncopal episodes in older patients. Arch Intern Med. 2009; 169(14):1299-1305. [PubMed: 19636031]

18. Johnson MR, Grubber J, Grambow SC, et al. Physician non-adherence to colonoscopy interval guidelines in the veterans affairs healthcare system. Gastroenterology. 2015; 149(4):938-951. [PubMed: 26122143]

19. Hsu JC, Chan PS, Tang F, Maddox TM, Marcus GM. Oral anticoagulant prescription in patients with atrial fibrillation and a low risk of thromboembolism: Insights from the NCDR PINNACLE registry. JAMA Intern Med. 2015; 175(6):1062-1065. [PubMed: 25867280]

20. January CT, Wann LS, Alpert JS, et al. 2014 AHA/ACC/HRS guideline for the management of patients with atrial fibrillation: Executive summary: A report of the american college of cardiology/american heart association task force on practice guidelines and the heart rhythm society. Circulation. 2014; 130(23):2071-2104. [PubMed: 24682348]

21. Jasuja GK, Bhasin S, Reisman JI, Berlowitz DR, Rose AJ. Ascertainment of testosterone prescribing practices in the VA. Med Care. 2015; 53(9):746-752. [PubMed: 26196850]

22. Baillargeon J, Urban RJ, Ottenbacher KJ, Pierson KS, Goodwin JS. Trends in androgen prescribing in the united states, 2001 to 2011. JAMA Intern Med. 2013; 173(15):1465-1466. [PubMed: 23939517]

23. Vigen R, O'Donnell CI, Baron AE, et al. Association of testosterone therapy with mortality, myocardial infarction, and stroke in men with low testosterone levels. JAMA. 2013; 310(17): 1829-1836. [PubMed: 24193080] 
24. Larochelle MR, Liebschutz JM, Zhang F, Ross-Degnan D, Wharam JF. Opioid prescribing after nonfatal overdose and association with repeated overdose: A cohort study. Ann Intern Med. 2016; 164(1):1-9. [PubMed: 26720742]

25. Dowell D, Haegerich TM, Chou R. CDC guideline for prescribing opioids for chronic pain - united states, 2016. MMWR Recomm Rep. 2016; 65(1):1-49.

26. Lipska KJ, Ross JS, Miao Y, Shah ND, Lee SJ, Steinman MA. Potential overtreatment of diabetes mellitus in older adults with tight glycemic control. JAMA Intern Med. 2015; 175(3):356-362. [PubMed: 25581565]

27. Lipska KJ, Krumholz H, Soones T, Lee SJ. Polypharmacy in the aging patient: A review of glycemic control in older adults with type 2 diabetes. JAMA. 2016; 315(10):1034-1045. [PubMed: 26954412]

28. Kirkman MS, Briscoe VJ, Clark N, et al. Diabetes in older adults: A consensus report. J Am Geriatr Soc. 2012; 60(12):2342-2356. [PubMed: 23106132]

29. Sussman JB, Kerr EA, Saini SD, et al. Rates of deintensification of blood pressure and glycemic medication treatment based on levels of control and life expectancy in older patients with diabetes mellitus. JAMA Intern Med. 2015; 175(12):1942-1949. [PubMed: 26502220]

30. Friedman BW, Dym AA, Davitt M, et al. Naproxen with cyclobenzaprine, oxycodone/ acetaminophen, or placebo for treating acute low back pain: A randomized clinical trial. JAMA. 2015; 314(15):1572-1580. [PubMed: 26501533]

31. Schappert SM, Burt CW. Ambulatory care visits to physician offices, hospital outpatient departments, and emergency departments: United states, 2001-02. Vital Health Stat. 2006; 13(159):1-66.

32. Friedman BW, Chilstrom M, Bijur PE, Gallagher EJ. Diagnostic testing and treatment of low back pain in united states emergency departments: A national perspective. Spine (Phila Pa 1976). 2010; 35(24):E1406-11. [PubMed: 21030902]

33. Polage CR, Solnick JV, Cohen SH. Toxin immunoassays and clostridium difficile infection-reply. JAMA Intern Med. 2016; 176(3):414-415. [PubMed: 26954051]

34. Durante C, Costante G, Lucisano G, et al. The natural history of benign thyroid nodules. JAMA. 2015; 313(9):926-935. [PubMed: 25734734]

35. Guth S, Theune U, Aberle J, Galach A, Bamberger CM. Very high prevalence of thyroid nodules detected by high frequency (13 MHz) ultrasound examination. Eur J Clin Invest. 2009; 39(8):699_ 706. [PubMed: 19601965]

36. Haugen BR, Alexander EK, Bible KC, et al. 2015 american thyroid association management guidelines for adult patients with thyroid nodules and differentiated thyroid cancer: The american thyroid association guidelines task force on thyroid nodules and differentiated thyroid cancer. Thyroid. 2016; 26(1):1-133. [PubMed: 26462967] 
1445 overuse manuscripts from systematic review of

PubMed and Embase and top medical journals +

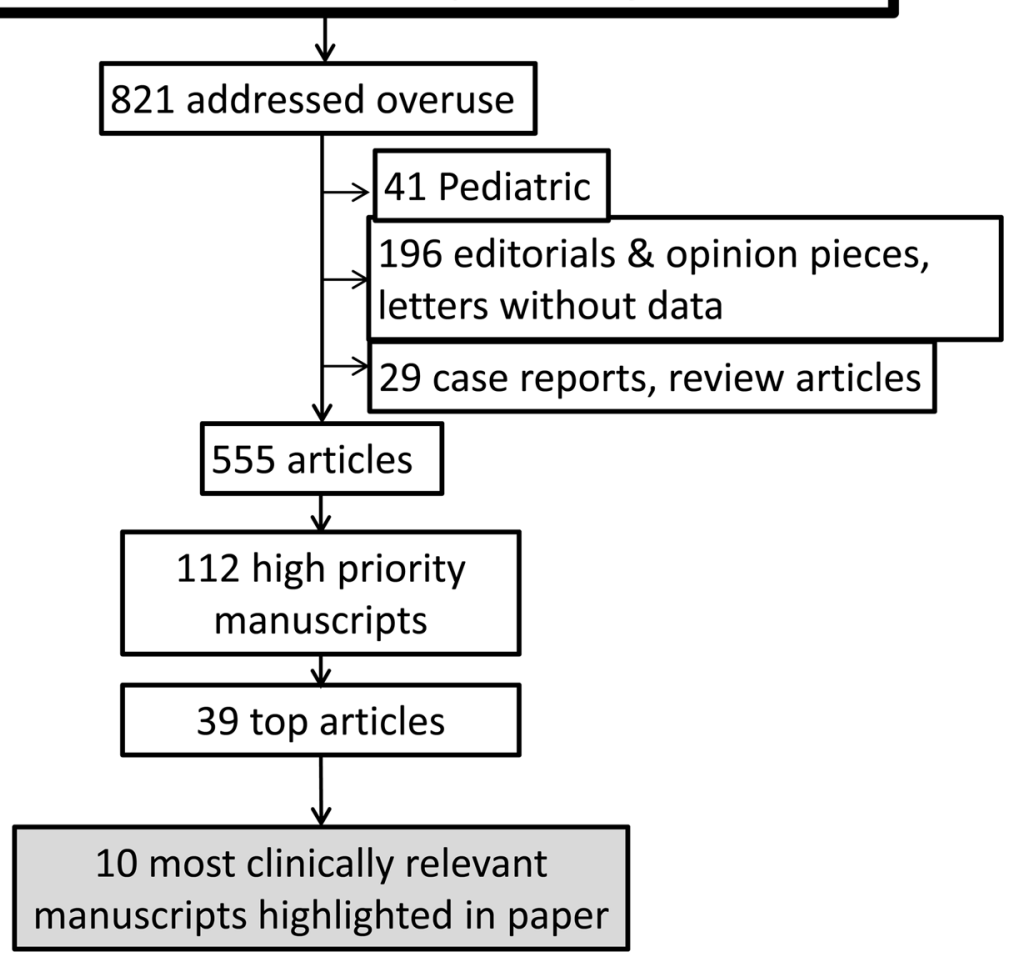

Figure 1. 